\title{
The Meaning of Debate and Critical Thinking to Foreign Language Education: A Cognitive Perspective
}

\author{
Zhong Deng \\ School of Foreign Languages and International Education \\ Hunan University \\ Changsha, China \\ Foreign Language School \\ Southwest Minzu University \\ Chengdu, China
}

\begin{abstract}
This paper is intended to elaborate on the theoretical and practical importance of debate and critical thinking to the foreign language education in China's higher learning institutions. By integrating the cognitive mechanisms responsible for critical thinking and those demanded by the teaching strategies for foreign language learners, this paper maintains that debate is an ideal form to be implemented in teaching plans as well as learning schemes for foreign language students regardless of their current language knowledge and skills. In this sense, this paper aims to argue for a shift of focus from the training of language skills to the fostering of critical thinking abilities on the part of foreign language students in China.
\end{abstract}

Keywords—debate; critical thinking; cognitive mechanisms; language students

\section{INTRODUCTION}

Language students in China's higher learning institutions have suffered from a prevalent point of view since the 1980s that the ultimate significance of such students lies in their language competence sufficiently qualified for occasions where communications demand clear and efficient renderings of both their mother tongue and foreign languages (translation ability, for example). Guided by such a conception of language education, language students have been criticized as lacking independent and critical thinking, thus failing in further intellectual development (Huang 1998). Confronted with such a criticism, a notion "Quality Education" was proposed in the 1990s. Vague as it may mean, this notion presents a departure from a rote-learning mode in the instruction of knowledge and skills and symbolizes an exploration for a revolutionary perception as well as methodology regarding what talents higher learning

This paper is supported by the Fundamental Research Funds for Central Universities. Project Title: A Cognitive Linguistics Approach to Chinese Abbreviations and their Meaning-Forming Mechanism (No. 2015SZYQN127). It is also supported by SMU Education Reform Project. Project Title: On the Improvement of Critical Thinking Competence of English Majors at SMU: Based on Courses of Intensive Reading (No. 2017YB09). institutions are supposed to produce and how to educate them. Despite controversies, an important consensus is that quality education is not the delivery of knowledge for the sake of knowledge itself, but a comprehensive process highlighting the capabilities of reasoning, integrating, comparing, questioning, inferring, abstracting and so forth (Trapp 2016). In other words, students are supposed to learn how to think instead of how to memorize knowledge points, which implies language education in China should experience a profound transformation from a knowledgeoriented model to a thinking-oriented one. However, given the wide acceptance of such a goal, there has been contentious argument on how to achieve it; this is especially true of language education, a realm that has been long perceived as emphasizing knowledge and skill acquisition only. Therefore, this paper, from the perspective of cognitive psychology and semantics, aims to contribute to the ongoing discussion concerning the methodology issue by pointing out that debate and the critical thinking behind it can be an ideal pattern to reach the ultimate goal in language education.

\section{DEBATE AND ITS COGNITIVE EsSENTIALS}

Debate may seem relatively alien for many Chinese teachers and students because, in many contexts, it has been traditionally mistaken for quarrel which Chinese people did not prefer as quarrel itself implies either absence of rationality or abuse of invective rhetoric. However, a closer investigation into what is going on in debate reveals that debate is a highly intellectually rewarding activity which motivates the participants to make efforts in many ways such as achieving a correct understanding of what is being debated, making a cogent argumentation for what is being upheld, launching a logical analysis of what opponents have argued and rendering a precise crystallization of what issues have been discussed and how the discussions are going (Wayne 1972). These aspects involved in debate are closely associated with the innate demand of language education, namely, giving top priority to thinking abilities. Such a close 
link can be well illustrated from a cognitive perspective as follows.

\section{A. Cognitive View of Meaning as the Base for Both Debate and Language Use}

A cognitive perspective to investigate human activities is primarily characterized by two theoretical assumptions. The first one is conceptualization. Viewed as a fundamental means for humans to make sense of the world, it is central to the working out of meaning of every object and experience concerned with day-to-day life (Talmy 2000). More precisely, meaning is considered the result of conceptualization (Langacker 2008). Another assumption, going further from conceptualization, is to state that meaning is encyclopedic. This statement implies that we cannot understand the meaning of any entity without considering the encyclopedic knowledge related to it (Langacker 2008). From the perspective of the embodiment philosophy (a philosophical foundation of cognitive linguistics, see Turner 1996), encyclopedic knowledge is the knowledge system constructed by the continuous interaction between human beings and the natural world as well as human societies. The knowledge system, including both the perceptual and rational cognition of a human body, alters and accumulates with the change of interactive space and time, thus forming and shaping the shared intellectual base of humans across nations and races.

But how is such a cognitive approach related to debate and language use? A clear link is that debate is about making sense of issues worth exchanges of opinions. In this sense, conceptualization means a cognitive path for people to the understanding of 1) what is the debate about? 2) What values or principles are behind the debate? 3) What issues are intrinsically related? 4) What arguments are involved? And more importantly, how are these questions cognitively related to the knowledge and critical thinking ability of individuals? Driven by these questions, we argue that conceptualization plays a key part in classifying, clarifying and crystallizing the input an individual learner has been offered in his educational and societal settings, while the encyclopedic processing of knowledge makes it possible for the learner to connect the knowledge from different sources of input into an integrated and controllable framework of schemas, which function as a two-way path facilitating abstraction from concrete substances and vice versa so as to guide debate in terms of monitoring meaning comprehension and expression for the sake of opinion exchanges. And the next step is the materialization of such conceptual schemas into language patterns (elaborated later in this chapter), which needs two procedures: 1) adopting appropriate vocabulary and grammatical structures to make understandable expressions and 2) making sure such expressions are among the most effective options for the purpose of arguing, analyzing or refuting when other choices are available as well.

\section{B. From Meaning to Language Patterning}

Conceptualization does not simply happen in people's brains; it is importance in debate and critical thinking is first of all embodied in the language patterning of speakers. In other words, conceptualization, elusive as it may seem, is materialized in observable patterning of language (especially in diction and syntactic/ discoursal arrangements) on the part of speakers who propose, refute and clarify arguments resulting from their making sense of the motion and the principles behind (see Talmy 2000). This way, we have actually identified a duality of conceptualization, the first level being central to meaning (linguistic and encyclopedic knowledge), and the second level being concerned with structure (grammatical patterning in light of cognitive operations). For example, we can find many debaters in a British Parliamentary (BP thereafter) debate frame are inclined to adopt such language patterns, though exact words and phrases may vary:

- The motion says.....and it really deals with....

- The aim of this policy is...

- Before constructing my case, I would like to make several rebuttals.

- You said...But it is a misunderstanding because....Therefore, we are doing a better job by arguing that...

Examples 1) and 2) are apparently concerned with a clarification of what is really being discussed, which calls upon the ability to identify the key words in the motion, to interpret the motion by activating the encyclopedic knowledge as the backcloth of conceptualization, and to connect the encyclopedic knowledge to the subsequent case to be constructed. Example 3) represents a cognitive endeavor of sequentialization, which means the speaker is well aware of the sequence of what should be done first and next, thus giving priority to what is more important. Besides, by looking at the speaker's differentiation of importance of tasks, we find rebuttals precede argument construction, which reveals a clarification process as Examples 1) and 2) illustrate. Example 4) is a rather challenging cognitive operation in that it aims to identify what is wrong with the opponent's opinions and why they are wrong. This operation calls for the capability of clarification, sequentialization, differentiation, and more importantly, elaboration. By elaboration, we mean the speaker cannot win by simply stating what is happening, but he or she has to shed a light on why is or is not the case by providing and processing concrete details. Thus, we may come up with a brief illustration on what is happening in debate and critical thinking: ation

clarification $\rightarrow$ sequentialization $\rightarrow$ differentiation $\rightarrow$ elabor

Not surprisingly, this illustration is not only represented by the language patterning adopted by debaters but also applicable to the language acquisition and development of language students as a whole. Therefore, we may hypothesize that the way of critical thinking is cognitively not distinguishable from the way of language acquisition, and it is the operational similarity between these two 
endeavors that illuminates how debate may and should fit in the intellectual development of language students.

\section{Why ForeIGN LANGUAGE ACQUISITION NEEDS CRITICAL THINKING}

Upon coming up with the above-mentioned hypothesis, we are obliged to prove its truthfulness and usefulness for language education in China. There are two tasks to complete: Firstly, we are to provide a detailed account of the cognitive mechanisms at work when students are involved in critical thinking; Secondly, we have to demonstrate how these cognitive mechanisms are inherently associated with language acquisition and development as underlying principles for the learning strategies of language learners as a whole.

\section{A. Clarification}

Basically, clarification is among the lowest-level cognitive mechanisms whose aim is literally to single out false elements from what is true. This involves what Langacker (2008) termed "construe", meaning an individualbased approach to understanding objects and concepts. According to Langacker (2008), the same conceptual content does not necessarily mean the same thing for different people; instead, it is the way individuals attempt to construe things that effectively determines what their understanding is like. This implies any individual is at least able to "frame" or "construct" something in their mind by means of figureground identification (Stockewell 2002), amplification of what draws attention (Talmy 2000), and mental integration (Turner 1996). The process of construal begins with semantic or semiotic decoding, followed by a cognitive connection of the decoded information to meaning analysis based on the cognizer's knowledge and perspective, and then the choice of the cognizer in terms of what to be figured or grounded, leading to a deliberate highlighting on what he or she is determined to comprehend. This process applies to both critical thinking and language learning, for both of them share the continuum of "input" (what is offered as a language point or a motion) and "intake" (what is comprehended). In language education, for instance, the "input" can involve a great variety of knowledge points; however, even though teachers believe all the language points are important and should be grasped, students may be guided by a set of criteria unconsciously so as to "take in" only what they believe more relevant. This process of singling out what is considered irrelevant or uninteresting is motivated by the students' cognitively driven choices based on their conceptualization of what is taught, thus functioning as feedback for the teachers to help them better know what is happening to students' learning activities.

\section{B. Sequentialization}

Sequentialization goes beyond clarification, but may occur simultaneously. It is associated with a cognitive mechanism called fictive scanning (Talmy 2000), which is responsible to explain how we decide to give priority to some certain points of information in language use. The principle is clear and crystal: our brain works like a scanner in preparation for constructing meaningful sentences by fictively "scanning" (occurring in the conceptual system, not in reality) the event(s) related and the components included. For example, when we say "the path winds up to the top of the hill", the path in reality is motionless; the point is that we initiate fictive scanning, i.e. we assume or picture humans' behavior of climbing up the hill via the path mentioned. This cognitive mechanism means lot to language acquisition because a) students need such a fictive activity to figure out precise uses of words and phrases especially when they are polysemic; and b) this activity contributes to students' production of expressions in unfamiliar settings. For example, when learning the word "see", a student is likely to come across such a sentence pattern: "The past decades saw a rapid development of..." In order to understand why "see" is used this way, the student may initiate a fictive activity in which he first of all personifies the subject "the past decades" into an animated agent, and then builds a semantic linkage between such an animated subject and the action of "saw", followed by the framing of another linkage between the verb "saw" and what is seen (as the object).

This cognitive process is indispensable for learners not only because it helps them understand words and expressions flexibly and correctly, but also because it allows the students to make rational decisions on what to emphasize or prioritize in sentence structuring so as to adopt the most effective pattern. In terms of learning the sentence pattern "It be (is/was...)...that...," there may be two choices for the students:

- The flight was delayed because of the bad weather.

- It was because of the bad weather that the flight was delayed.

Sentence a) simply depicts or reports a fact and the reason behind, while sentence b) prioritizes the reason for what was happening. In this case, students are confronted with a task of choosing a sentence pattern in accordance with what information they perceive is more urgent, i.e. they have to fictively scan what the purpose of the communication is, what has been said and has happened so far, and what information is expected for the hearers. This is in effect concerned with a cognitive pragmatic investigation which is also based on the cognitive mechanisms we are discussing.

\section{Differentiation}

Differentiation is among relatively more demanding cognitive mechanisms, which plays an irreplaceable part in facilitating students to project what is more semantically salient or important in an utterance or sentence onto appropriate grammatical structures, especially when two or more structures are semantically similar to one another. The core of differentiation is to establish a mental framework as to in what way similar language items are different or vice versa and how to explain such a relatively dynamic state of similarity and difference. In language acquisition, this framework is embodied by the perception of meaning and form paring (Langacker 2008). A pertinent example is the acquisition of voice of English sentences. Traditionally, the active voice has not been deliberately differentiated from the 
passive voice as in the sentences a) "300 workers build the mansion." and b) "The mansion is built by 300 workers." Teachers have agreed on the semantic sameness of these two sentences because according to typical Chomskyan elaboration (1957), the structural transformation from a) to b) is merely concerned with the level of the surface structure, while the deep structure remains the same. However, students may come across some unexpectedly confusing examples if they are instructed to make such a transformation practice. For instance, they may doubt the semantic sameness when they look at c) "Beavers build dams." and d) "Dams are built by beavers." No doubt, sentences c) and d) represent an active-to-passive voice transformation as is shown by sentences a) and b), but the meaning of c) is not necessarily the same with that of d): c) talks about one of the living habits of beavers, while d) deals with how dams are constructed (Liu \& Ai 2016). Therefore, what the teachers have instructed becomes somewhat invalid. In this case, differentiation as a cognitive operation can be called upon. In other words, students have to distinguish between these two scenarios by understanding what is happening in the two cases respectively and more desirably, figuring out what reasons are held accountable for such a difference. To help students achieve this, teachers are advised to remind the students of the importance of coping with confusing uses of familiar language patterns, instead of getting accustomed to what the textbooks have prescribed.

\section{Elaboration}

One of the most rewarding objectives of language acquisition is to gain the ability to elaborate and convince. And this ability is associated with the cognitive operation termed elaboration. In day-to-day use of English, "elaboration" is usually a concrete act of providing details and analyses for arguments, but cognitively speaking, this term means more of a mental exploration than observable behavior because it is directly relevant to the notion of "specificity" proposed by Langacker (2008), which, as an important dimension in accounting for people's construal of entities, has two implications: Firstly, as has been discussed above, different people take different perspectives to look at and understand entities. Secondly, as to in what way one's perspective is different from that of others, Langacker identified that the degree to which people specify an entity is among the most remarkable variables. In other words, some people usually do not pursue detailed specifications in construing objects; for example, they may know the creature they see flying is a bird, but they do not bother themselves to ask what exactly that bird is. On the contrary, other people may have developed a habit of seeking details as many and relevant as possible to facilitate their construal of entities; for instance, when giving oral guidance to those who ask for directions, they can be very careful and patient on the specificity and credibility of what they say, as if they were living maps. In language education, accordingly, students are faced with the problem of whether they are able to provide reasonably acceptable specifications in their language use. More specifically, this problem is related to two subconcerns: 1) whether it is always necessary to specify entities in terms of vocabulary and structures (actually an issue connected to "sequentialization") and 2) how they should specify entities when necessary. For the latter concern, there are several suggestions for both teachers and students.

In vocabulary teaching, the core task therefore is to attach more importance to the hierarchy of word meaning (hyponymy). Take "bird" for example, such a "big" word may not be desirable in many contexts especially when students are required to write descriptive sentences or articles on the natural environments and the wildlife. Thus, it is recommended that teachers help students build a conceptual network of hierarchical meanings of words. On sentence level, students are expected to become aware that some patterns are more powerful in terms of representing detailed and well-illustrated information such as the "with+NP" and "V+ing" constructions as adverbials. Besides, they need know the appropriate use of subordinated clauses also increases the degree of specificity of sentences while helping present a rational arrangement of information in the meantime. As for discourse construction, the most important training is to ask the students to provide concrete, detailed, logically-arranged elaborations for what they narrate, describe or argue. This include their cognitive awareness of drawing on their own experience, citing statistics and examples when needed, and extending existing details or evidence by seeking their potential application to other realms.

\section{CONCLUSION}

Debate and critical thinking are not simply intellectual activities or training programs colleges and universities may choose to facilitate students to increase their knowledge or enhance the appeal of their on-campus academic events. From a cognitive perspective, instead, this paper maintains that the underlying cognitive mechanisms are tremendously important for foreign language education in China as they depart from the traditional practices of teaching and learning and highlight the internally motivated endeavor of the learners by activating their cognitive operations for language acquisition and processing at vocabulary, sentence and discourse levels. Besides, the cognitive approach is intrinsically related to the pragmatic concern of language, which helps students produce appropriate utterances for varieties of situations. Meanwhile, teachers also benefit from this cognitive point of view as they may have more tangible feedback from the students and they are driven to adjust their teaching methods to cope with such feedback. Last but not least, debate itself serves a common form for the students to practice their language skills, though somewhat demanding for beginners. In conclusion, this paper appeals for a shift from focusing on the pedagogical training of language knowledge and skills to fostering critical thinking abilities on the foreign language students in China.

\section{REFERENCES}

[1] B. Wayne. "Arguers as Lovers," Philosophy and Rhetoric. (5) 1972:1-11.

[2] E. Douglas. Decision by Debate. 10th ed. New York: Dodd Mead, 1963. 
[3] L. Talmy. Toward a Cognitive Semantics, Vol II: Typology and Process in Concept Structuring. Cambridge/Massachusetts: The MIT Press, 2000.

[4] M. Turner. The Literary Mind: The Origins of Language and Thought. Oxford: Oxford University Press, 1996.

[5] N. Chomsky. Syntactic Structures. The Hague: Mouton, 1957.

[6] R. Langacker. Cognitive Grammar: A Basic Introduction. Oxford: Oxford University Press, 2008.

[7] R.Trapp et al. Building Global Relations through Debate. Beijing: Foreign Language Teaching and Research Press, 2016.

[8] Huang Yuanshen. The Absence of Critical Thinking. Foreign Languages and Their Teaching, 1998 (7):1.

[9] Liu Zhengguang, Ai Zhaoyang. A Cognitive Linguistics Approach to the Fundamental Issues of Foreign Language Teaching. Linguistics and Applied Linguistics, 2016 (2): 257-266. 